\title{
Reading comprehension of health checkup reports and health literacy in Japanese people
}

\author{
Machi Suka $\cdot$ Takeshi Odajima $\cdot$ Masako Okamoto • \\ Masahiko Sumitani • Takeo Nakayama • \\ Hiroki Sugimori
}

Received: 21 February 2014/ Accepted: 7 April 2014/Published online: 9 May 2014

(c) The Japanese Society for Hygiene 2014

\begin{abstract}
Objectives To determine the reading comprehension of health checkup reports in the context of health literacy (HL) in Japanese people.

Methods A web-based survey was conducted among 424 Japanese adults aged 35-59 years. Participants were asked to read specifically designed health checkup reports and then answer a series of questions to examine whether they accomplished the fundamental purposes of health checkup reports (recognition of the problems, recognition of the risk of illness, recognition of the need for preventive action, and motivation for preventive action). HL was simultaneously
\end{abstract}

M. Suka $(\bowtie)$

Department of Public Health and Environmental Medicine, The Jikei University School of Medicine, 3-25-8 Nishi-

Shimbashi, Minato-ku, Tokyo, Japan

e-mail: suka@jikei.ac.jp

T. Odajima

Japanese Red Cross Kanto-Koshinetsu Block Blood Center, Tokyo, Japan

M. Okamoto

Department of Applied Biological Chemistry, Graduate School of Agricultural and Life Sciences, The University of Tokyo, Tokyo, Japan

\section{Sumitani}

Department of Pain and Palliative Medicine, The University

of Tokyo Hospital, Tokyo, Japan

T. Nakayama

Department of Health Informatics, Kyoto University

School of Public Health, Kyoto, Japan

H. Sugimori

Department of Preventive Medicine, Graduate School of Sports and Health Sciences, Daito Bunka University, Saitama, Japan measured using the 14-item health literacy scale (HLS-14), the 11-item Lipkus scale (Lipkus-J), and the Newest Vital Sign (NVS-J).

Results About $70 \%$ of the study subjects misread the normal/abnormal classification for at least one items. Those with lower HLS-14 scores were significantly less likely to recognize the problems, the risk of illness, and the need for preventive action for the examinee, and also less likely to express their willingness to take preventive action in compliance with the doctor's advice after having received the health checkup report. Compared with the HLS-14 scores, the Lipkus-J and NVS-J scores showed hardly any association with the reading comprehension of health checkup reports.

Conclusion All examinees do not always have an adequate level of HL. HL may be the major determinant of reading comprehension of health checkup reports. For more effective health checkups, health promotion service providers should become aware of the existence of examinees with inadequate $\mathrm{HL}$ and address the problem of misreading health checkup results.

Keywords Reading comprehension - Health checkup report · Health literacy · Japan

\section{Introduction}

Multiphasic health checkups are annually conducted according to the law in community and worksite in Japan. Every examinee receives a piece of paper that conveys his/ her health checkup results within a few months after the health checkup. Health promotion service providers expect the examinees to read their health checkup reports and act on the doctor's advice written there. Although Japan has 
one of the most educated populations in the world [1], well-educated people do not always have an adequate level of health literacy (HL). It may be more complicated that it looks for ordinary (non-medical) people to understand their health checkup reports that notify measurement data and professional comment with a lot of technical jargons. Unfortunately, epidemiological evidence to support the reading comprehension of health checkup reports has been scarce so far.

To what extent people seek, understand, and use health information depends on their HL level [2, 3]. Recently, a number of instruments have become available to measure HL in Japanese speakers [4-9]. In the absence of a gold standard, composition and content vary widely across these instruments, and they seem to measure different constructs of HL. There have been no studies that simultaneously measured HL using two or more instruments in a Japanese population. Accordingly, little is known about the discrepancy between existing measures of HL.

To produce the effect expected from health checkups, health checkup results must be notified in a way that is understandable to all examinees regardless of their $\mathrm{HL}$ level. It is important to determine the reading comprehension of health checkup reports in the context of HL in a possible target population. We conducted a web-based survey among middle-aged Japanese people to investigate to what extent they could read specifically designed health checkup reports [10]. HL was simultaneously measured using the following three instruments: the 14-item health literacy scale (HLS-14) [6], the 11-item Lipkus scale (Lipkus-J) [7], and the Newest Vital Sign (NVS-J) [8]. These instruments, consisting of 14, 11, and 6 questions, respectively, capture different aspects of $\mathrm{HL}$ and enable users to obtain a single index value for HL. Moreover, these instruments are designed for self-completion by respondents and are ideally suited for use in both paperbased and web-based surveys. In this study, we analyzed the relationship between the reading comprehension of health checkup reports and the three types of HL measures in Japanese people.

\section{Methods}

\section{Subjects}

A web-based survey was conducted in June 2013 among 424 Japanese adults aged 35-59 years [10]. An online research company (MACROMILL, INC., Tokyo, Japan) contracted to create web questionnaire forms and collect responses $(n=400)$. The company has a nationwide research panel of more than 1 million registrants. At the time of the survey, the registrants aged 35-59 years totaled
534,582 (221,815 males and 312,767 females). Recruitment e-mails were sent to 20,000 eligible registrants aged 35-59 years who were randomly selected from each age and gender stratum. Applicants for participation in the survey were accepted in the order of receipt until the number of participants reached the quotas (40 people aged $35-39$ years, 80 people aged $40-49$ years, and 80 people aged 50-59 years for each gender). All participants voluntarily agreed to complete the survey. Those with serious health conditions and medical professionals were excluded through a prescreening process. Finally, a total of 424 valid responses were obtained. The study protocol was approved by the ethics committees of the Jikei University School of Medicine and has been conducted in accordance with the Guidelines for Epidemiological Studies by the Ministry of Health, Labour, and Welfare and the Ministry of Education, Culture, Sports, Science, and Technology.

Measures

\section{Health literacy}

HL was simultaneously measured using the HLS-14, the Lipkus-J, and the NVS-J. These instruments were developed to measure different constructs of $\mathrm{HL}$ and were validated in Japanese people [6-8].

The HLS-14 [6] consists of 5 items for functional HL, 5 items for communicative HL, and 4 items for critical HL. The functional HL items ask about basic skills to read instructions or leaflets from hospitals or pharmacies. The communicative HL items asked about more advanced skills to obtain information about disease and treatment and use the obtained information in everyday situations. The critical HL items asked about more advanced skills to analyze information about disease and treatment critically. Respondents choose one of 5 options (1: strongly agree/disagree to 5 : strongly disagree/agree) in response to each statement. The scores on the items were summed up to give the HLS-14 score (range 14-70 points) for each respondent.

The Lipkus-J [7, 11] consists of 3 general numeracy questions to measure understanding of percentages, frequency, and probability and 8 expanded numeracy questions to assess the ability to comprehend numerical risk information that are represented as percentages, frequencies, and probabilities in medical scenarios. For each question, respondents perform a simple calculation and answer the calculation result by means of giving figures or choosing one of the options. The number of the correct answers was counted as the Lipkus-J score (range 0-11 points) for each respondent.

The NVS-J $[8,12]$ is designed to assess the ability to understand and act on health information. Subjects are given a specially designed ice cream nutrition label and 
are asked 6 questions about it. For the first four questions, respondents derive relevant information from the nutritional label and perform a simple calculation to get a specific numerical answer. For the fifth question, respondents judge whether a person allergic to peanuts can eat the ice cream, referring to the nutrition label, and for the sixth question, they explain the reason for the judgment. The number of the correct answers was counted as the NVS-J score (range 0-6 points) for each respondent.

Higher scores indicate having better HL for all measures.

Reading comprehension of health checkup reports

There is no established methodology for assessing reading comprehension of personal health information like health checkup reports. In this study, participants were asked to read specifically designed health checkup reports and then answer a series of questions to examine whether they accomplished the fundamental purposes of health checkup reports.

\section{Preparation of health checkup report examples}

Prior to the web-based survey, we collected health checkup report samples from 20 healthcare facilities throughout Japan. Based on the review of health checkup report samples, we found five distinctive features of health checkup reports and then prepared five representative examples for the web-based survey [10].

Participants in the web-based survey were randomly divided into four groups (53 males and 53 females, respectively). Each group was assigned two of the five health checkup report examples: one example was a standard and another example had some different features which may be influential in reading the health checkup report. There were two kinds of health checkup report examples that were considered as the standard. These health checkup report examples had the same form while they were applied different coding systems (alphabet grades vs. Japanese grades). The health checkup report example with alphabet grades is shown in Fig. 1. The health checkup report example with Japanese grades looked the same as that of the Fig. 1, except that the grades were expressed in the Japanese codes. Two groups were assigned the health checkup report example with alphabet grades as a standard (presented in different orders) and the other two groups were assigned the health checkup report example with Japanese grades as a standard (presented in different orders). When we preliminarily compared the answers to the questions about the health checkup report example with alphabet grades and those of the health checkup report example with Japanese grades, no significant differences were found between them. Moreover, there were no significant differences between the two groups in which the same health checkup report example was presented as a standard in different orders (i.e., order effects). Therefore, in this study, the answers to the questions about the health checkup report example with alphabet grades and those of the health checkup report example with Japanese grades were combined and analyzed together.

As shown in the Fig. 1, the health checkup report example consisted mainly of findings and advice. A hypothetical case of metabolic syndrome was embedded in every health checkup report example. Physical measurements and clinical laboratory data were listed on the right side of the document. Abnormal findings were pointed out in the following 7 items: (1) body composition (waist circumference $87.5 \mathrm{~cm}$ ) graded C or 'follow-up required', (2) blood pressure (under treatment) graded $\mathrm{F}$ or 'under treatment', (3) liver function (alanine aminotransferase 46 $\mathrm{U} / \mathrm{l}, \gamma$-glutamyl transferase $81 \mathrm{U} / \mathrm{l}$ ) graded $\mathrm{C}$ or 'follow-up required', (4) serum lipid (triglyceride $369 \mathrm{mg} / \mathrm{dl}$, HDL cholesterol $37 \mathrm{mg} / \mathrm{dl}$ ) graded C or 'follow-up required', (5) blood glucose (hemoglobin A1c $6.3 \%$ ) graded D or 'precise reexamination required', (6) uric acid (uric acid $7.1 \mathrm{mg} / \mathrm{dl}$ ) graded C or 'follow-up required', and (7) electrocardiogram (left ventricular hypertrophy) graded C or 'follow-up required'. A doctor's advice on the abnormal findings was written in the text box on the left side of the document. The doctor recommended the examinee to improve current lifestyle, especially diet and alcohol; to continue antihypertensive therapy; to have a precise reexamination for blood glucose; to have a 6-month follow-up examination for serum lipid; and to have a regular checkup next year to monitor the items that were classified as abnormal in this health checkup report. Overall, the health checkup results indicated that the examinee held multiple metabolic risk factors, which is called metabolic syndrome, and thus had to take appropriate measures to minimize his risk of illness.

\section{Assessment of reading comprehension}

Generally, health checkup reports are served on examinees for the purpose of notifying them what problems they have (recognition of the problems), to what extent their problems may induce seriously ill (recognition of the risk of illness), and what measures they must take to minimize their risk of illness (recognition of the need for preventive action), and consequently encouraging their willingness to take preventive action in compliance with their doctor's advice (motivation for preventive action). In this study, the assessment of reading comprehension 


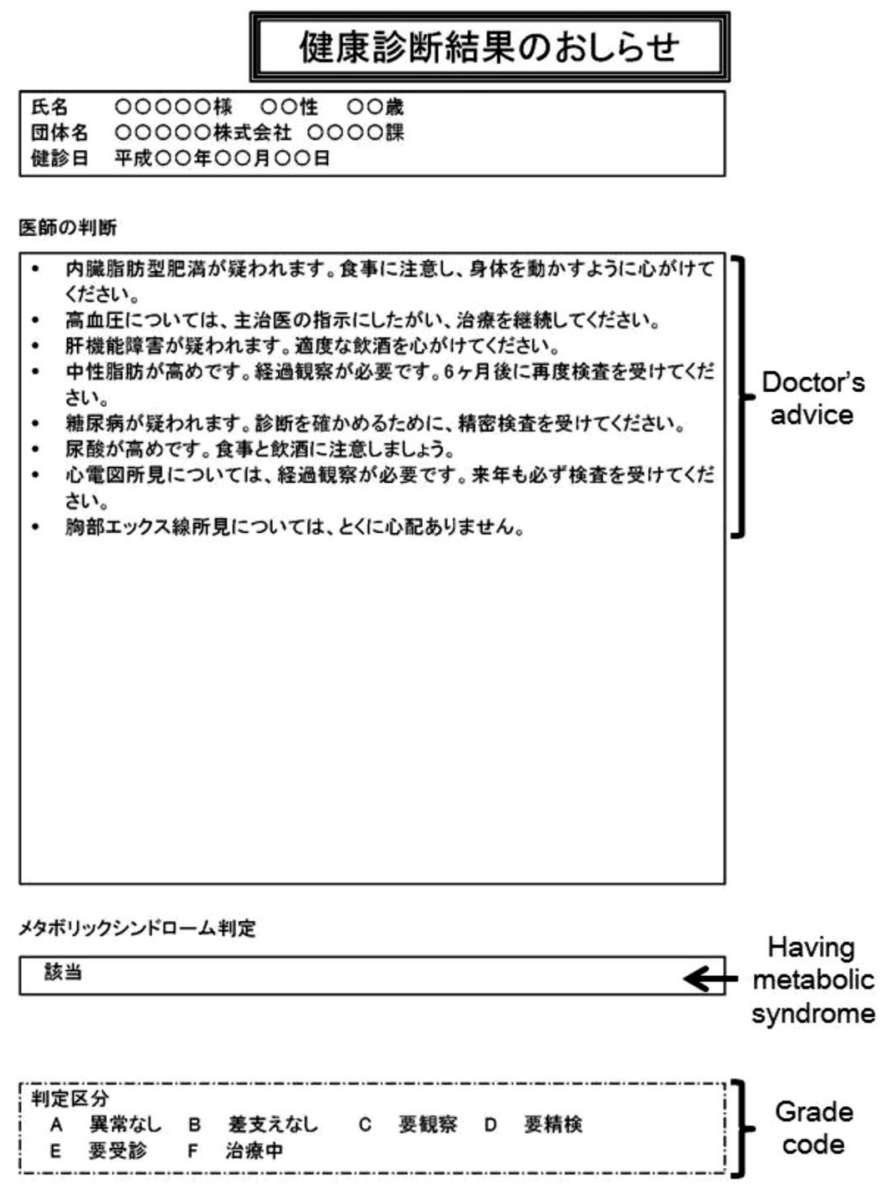

Fig. 1 Health checkup report example used in the web-based survey

focused on addressing these fundamental purposes of health checkup reports.

To examine whether participants accomplished the first purpose (recognition of the problems), they were asked to point out all the items that were classified as abnormal in the health checkup report. A list of 12 items was displayed and corresponding items were checked. As for the second and third purposes (recognition of the risk of illness and the need for preventive action), participants were asked to rate how much they agree or disagree with the following statements on a 5-point scale: I think that this examinee (1) has an increased risk of illness, (2) will get seriously ill unless he takes measures, (3) will recover his health if he takes measures, and (4) should take appropriate measures. As for the fourth and final purpose (motivation for preventive action), participants were asked to rate how much they agree or disagree with the following statements on a 5-point scale: If I were this examinee and received this health checkup report, I would (1) have a precise

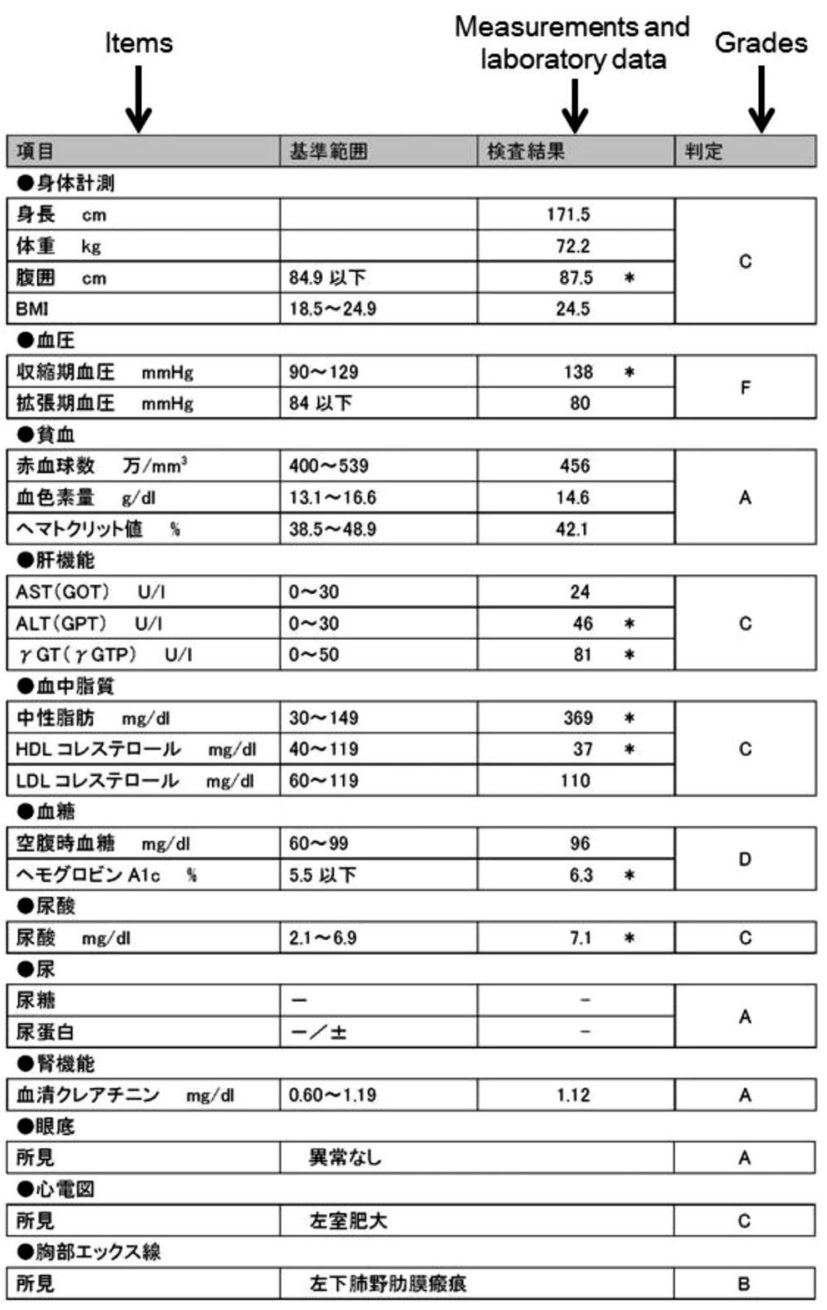

reexamination, (2) have a 6-month follow-up examination, (3) improve current lifestyle, and (4) have a regular checkup next year.

Participants were also asked to assess the comprehensibility of the health checkup report. According to the consumer information rating form [13], participants rated how easy or hard the health checkup report was to (1) read, (2) understand, (3) remember, (4) locate the point, and (5) keep for future reference on a 5-point scale.

Statistical analysis

Median and interquartile range (IQR) were calculated for the HLS-14, Lipkus-J, and NVS-J scores and were compared using Mann-Whitney test (between two groups) or Kruskal-Wallis test (between three or more groups). Spearman correlation coefficients $(\gamma)$ were calculated to determine the associations between the HLS14, Lipkus-J, and NVS-J scores. To examine whether the 
HLS-14, Lipkus-J, and NVS-J scores were related to the reading comprehension of health checkup reports, the study subjects were divided into two groups according to the HLS-14, Lipkus-J, and NVS-J scores above or below the median, respectively. Responses were recategorized into three groups for comparison: strongly disagree/disagree, uncertain, and agree/strongly agree; very difficult/ difficult, neutral, and easy/very easy. The distribution of responses was compared between the higher and lower scoring groups using Chi-square test. All statistical analyses were performed using the SAS ver. 9.2 (SAS Institute, Cary, NC, USA). Significant levels were set at $p<0.05$.

\section{Results}

\section{Subjects}

Table 1 shows the characteristics of the study subjects. Of the 424 subjects, $46.7 \%$ had completed higher education (university or higher), $68.9 \%$ were married, and $73.8 \%$ had some occupation. Those who reported having a health checkup every year accounted for $62.0 ; 10.1 \%$ had every 2 years, $5.7 \%$ had every 3-4 years, and $22.1 \%$ had every 5 years or less. According to the 2011 national census [14], the percentage of the Japanese population aged 35-59 years with university degrees was $21.3 \%$, considerably lower than that of this study, whereas the percentages of married and employed population were 71.3 and $74.8 \%$, respectively, almost equal to that of this study.

\section{Health literacy}

Figure 2 shows the distribution of the HLS-14, Lipus-J, and NVS-J scores. The HLS-14 scores were approximately normally distributed with a median of 50 (IQR 10) points. Higher HLS-14 scores were associated with being female $(p<0.001)$, being more highly educated $(p<0.001)$, having no occupation $(p<0.001)$, and having good economic status $(p=0.002)$. The distribution of the Lipkus-J scores was left-skewed with a median of 10 (IQR 2) points; $47.1 \%$ of the study subjects got a perfect score. Higher Lipkus-J scores were associated with being male ( $p<0.001)$, being older $(p=0.006)$, being more highly educated $(p<0.001)$, and being an employee $(p=0.008)$. The distribution of the NVS-J scores was left-skewed with a median of 4 (IQR 2) points. Higher NVS-J scores were associated with being older ( $p=0.034)$, being more highly educated $(p=0.028)$, and having good economic status $(p=0.007)$.

When the associations between these three measures were examined, the Lipkus-J scores and the NVS-J scores
Table 1 Characteristics of the study subjects

\begin{tabular}{|c|c|c|}
\hline & $n$ & $\%$ \\
\hline \multicolumn{3}{|l|}{ Age } \\
\hline 35-39 years & 88 & 20.8 \\
\hline $40-44$ & 92 & 21.7 \\
\hline $45-49$ & 76 & 17.9 \\
\hline $50-54$ & 99 & 23.3 \\
\hline $55-59$ & 69 & 16.3 \\
\hline \multicolumn{3}{|l|}{ Gender } \\
\hline Male & 212 & 50.0 \\
\hline Female & 212 & 50.0 \\
\hline \multicolumn{3}{|l|}{ Education } \\
\hline Compulsory education & 10 & 2.4 \\
\hline High school & 119 & 28.1 \\
\hline Junior college/vocational school & 96 & 22.6 \\
\hline University or higher & 198 & 46.7 \\
\hline Missing & 1 & 0.2 \\
\hline \multicolumn{3}{|l|}{ Marital status } \\
\hline Unmarried & 132 & 31.1 \\
\hline Married & 292 & 68.9 \\
\hline \multicolumn{3}{|l|}{ Occupational status } \\
\hline No occupation & 111 & 26.2 \\
\hline Self-employed & 75 & 17.7 \\
\hline Temporary or part-time employee & 60 & 14.2 \\
\hline Regular full-time employee & 178 & 42.0 \\
\hline \multicolumn{3}{|l|}{ Household income } \\
\hline$<2.0$ million yen $^{\mathrm{a}}$ & 31 & 7.3 \\
\hline $2.0-5.9$ & 176 & 41.5 \\
\hline $6.0 \leq$ & 166 & 39.2 \\
\hline Missing & 51 & 12.0 \\
\hline \multicolumn{3}{|l|}{ Self-rated economic status } \\
\hline Very poor & 97 & 22.9 \\
\hline Poor & 116 & 27.4 \\
\hline Moderate & 134 & 31.6 \\
\hline Good & 77 & 18.2 \\
\hline
\end{tabular}

a 1 million yen was about 9,000 US dollars at the time of the survey

were moderately correlated with each other $(\gamma=0.42$, $p<0.001)$. Meanwhile, the HLS-14 scores showed significant but weak correlations with the Lipus-J scores $(\gamma=0.21, p<0.001)$ and the NVS-J scores $(\gamma=0.21$, $p<0.001)$.

Reading comprehension of health checkup reports

Table 2 shows the recognition of the problems. For the 7 items classified as abnormal, 25.0-52.4\% of the study subjects, respectively, misread the normal/abnormal classification, whereas for the 5 items classified as normal, almost all (93.9-99.1\%) gave the right answers. Overall, 
(a)

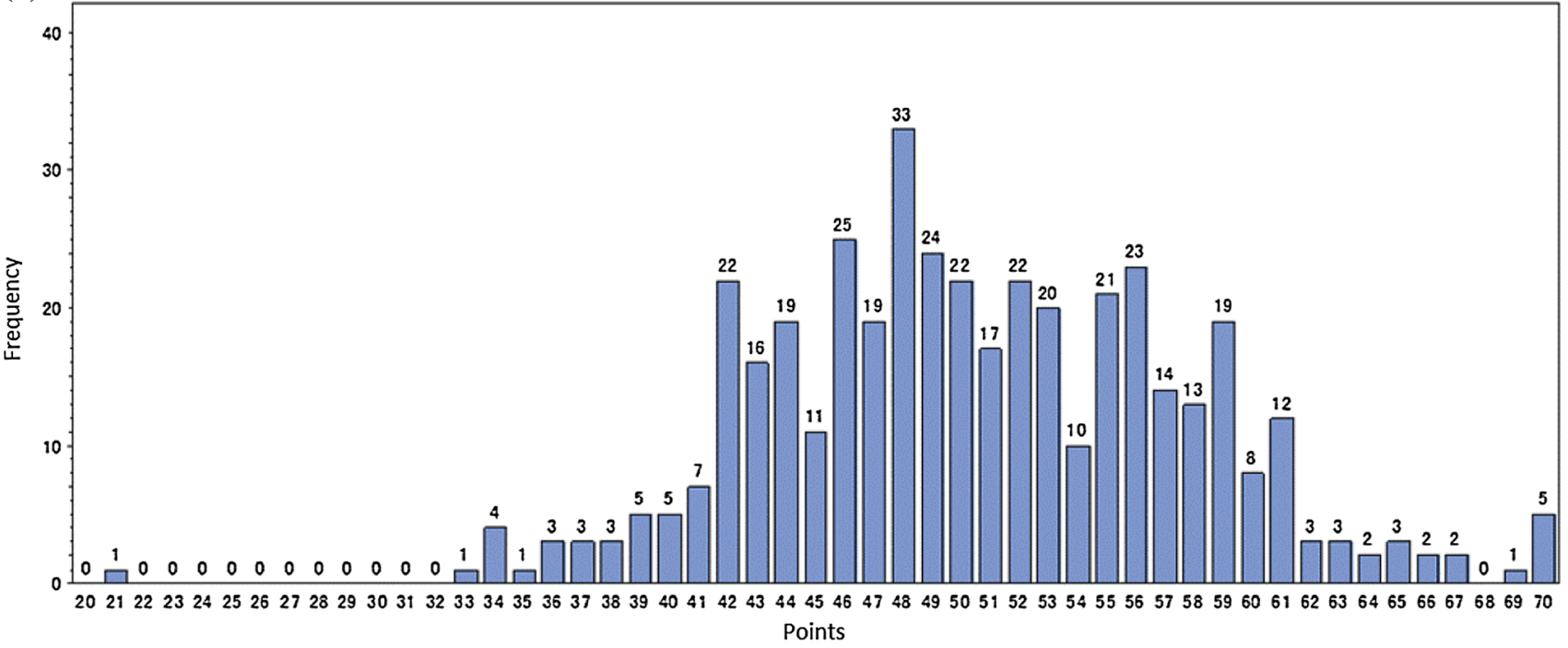

(b)

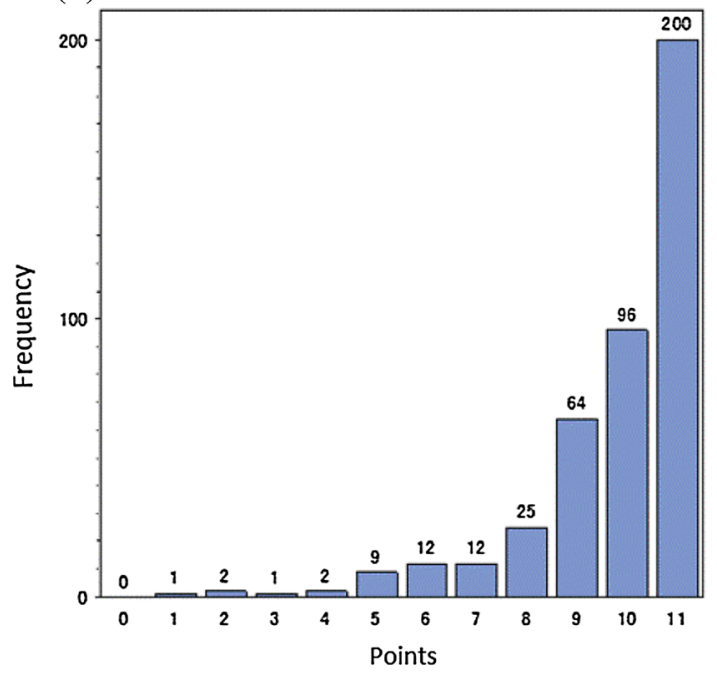

(c)

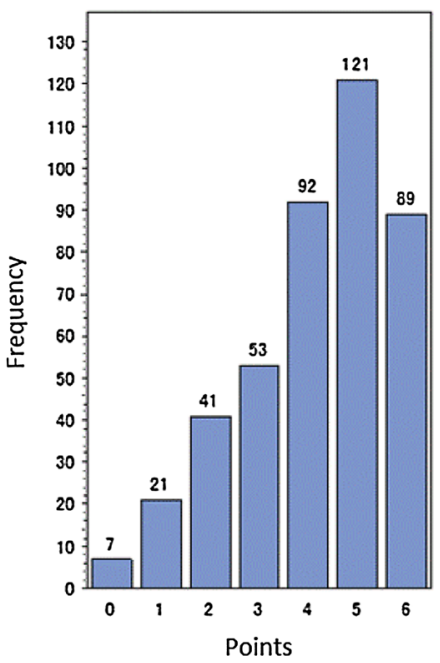

Fig. 2 Distribution of the HLS-14 (a), Lipkus-J (b), NVS-J (c) scores

those who could correctly point out all the items classified as abnormal accounted for $27.1 \%$ in the study subjects. This percentage was $36.5 \%$ in the HLS-14 higher scoring group compared with $18.8 \%$ in the lower scoring group $(p<0.001) ; 33.0 \%$ in the Lipkus-J higher scoring group compared with $21.9 \%$ in the lower scoring group $(p=0.010) ; 36.7 \%$ in the NVS-J higher scoring group compared with $17.8 \%$ in the lower scoring group $(p<0.001)$.

Table 3 shows the recognition of the risk of illness, the recognition of the need for preventive action, and the motivation for preventive action. As for the first 2 statements assessing their recognition of the risk of illness, 77.6 and $78.3 \%$ of the study subjects, respectively, agreed; whereas 11.1 and $10.4 \%$, respectively, expressed disagreement. As for the second 2 statements assessing their recognition of the need for preventive action, 73.8 and $74.1 \%$ of the study subjects, respectively, agreed; whereas 9.4 and $9.4 \%$, respectively, expressed disagreement. As for the latter 4 statements assessing their motivation for preventive action, those who expressed their willingness to have a precise reexamination, to have a 6-month follow-up examination, to improve current lifestyle, and to have a regular checkup next year in compliance with the doctor's advice accounted for $64.2,61.6,73.8$, and $83.0 \%$, respectively, in the study subjects. These percentages in the HLS-14 higher scoring group were significantly higher than those in the lower scoring group. As for the Lipkus-J, there were no significant differences between the higher and lower scoring groups. As for NVS-J, a significant 


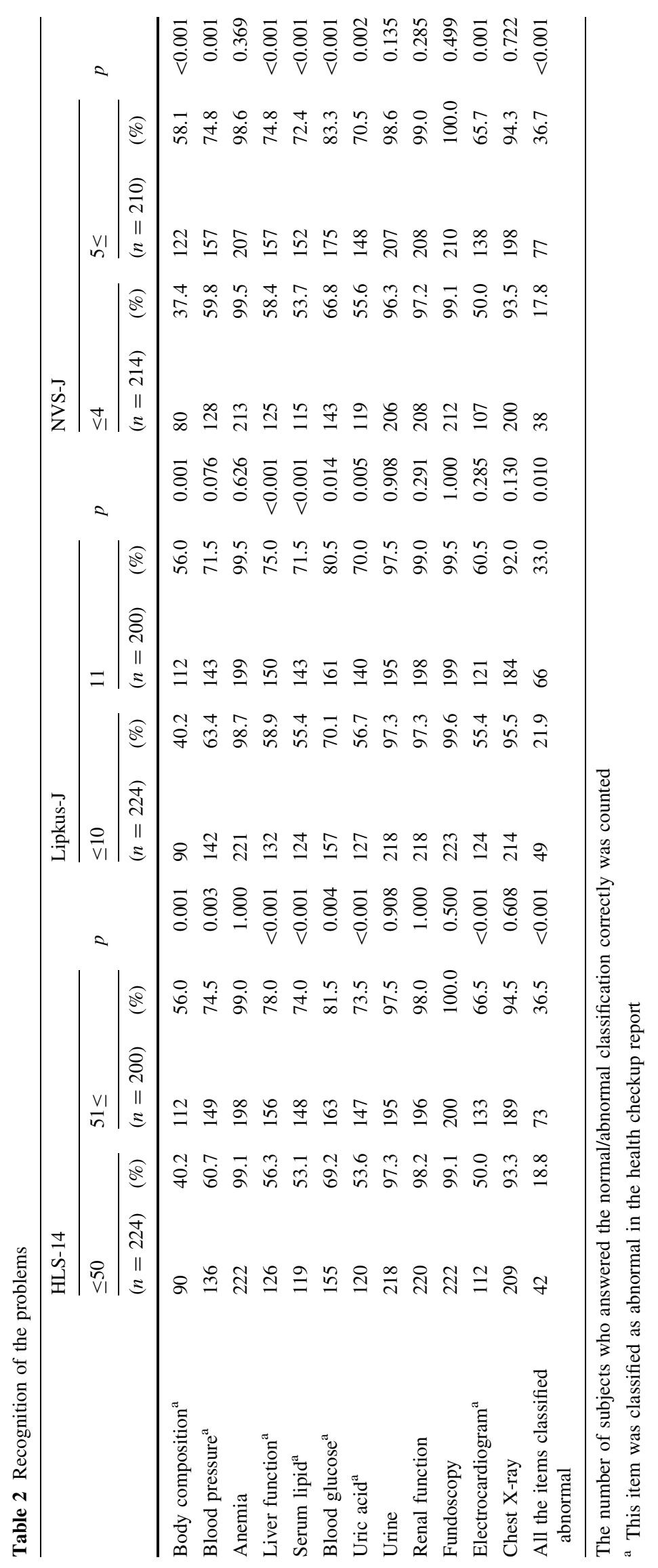




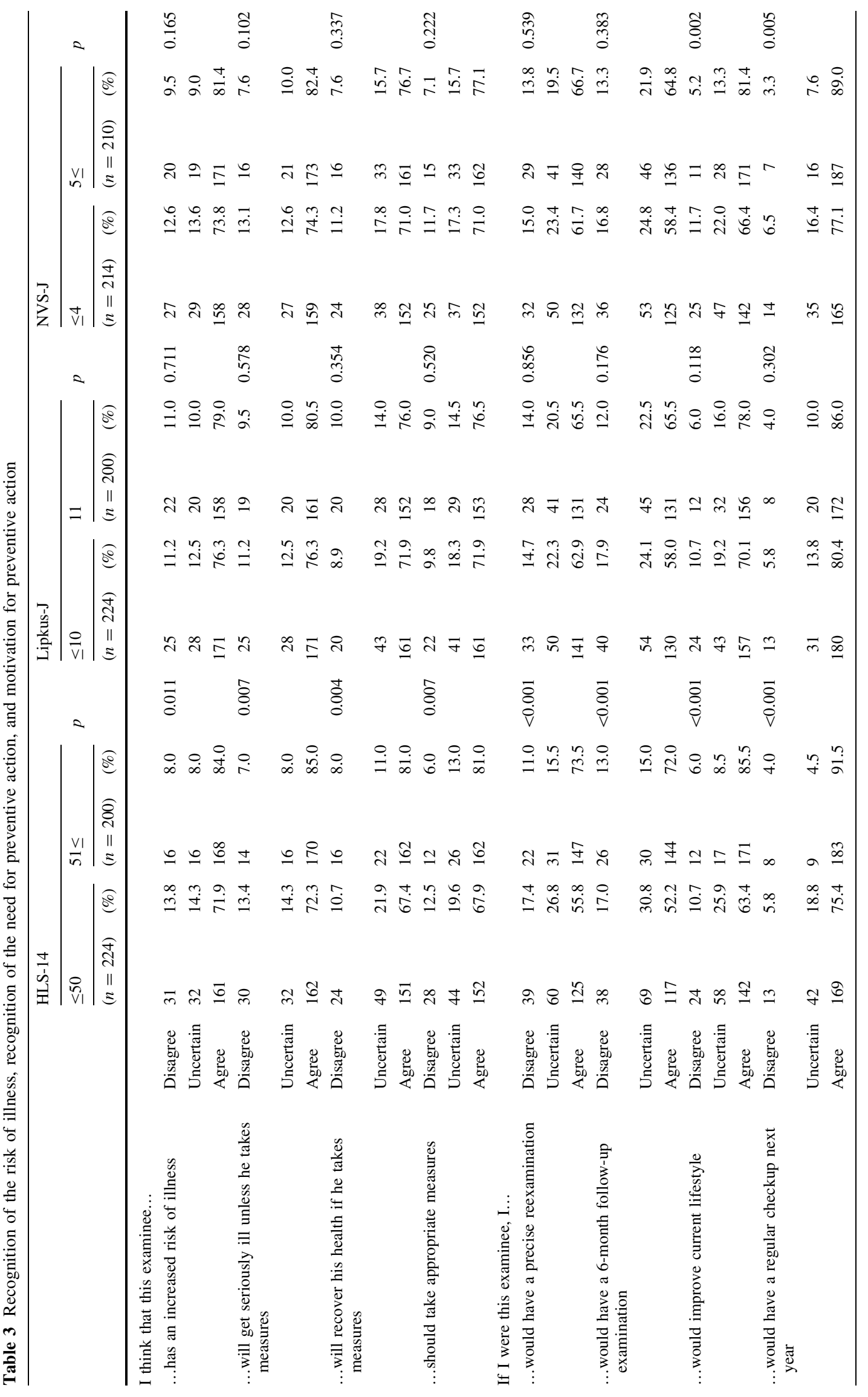


difference between the higher and lower scoring groups was found in the willingness to improve current lifestyle and have a regular checkup next year.

We further examine the relationship between the reading comprehension of health checkup reports and the HLS-14 scores by gender and by age group. Although the number of subjects was too small to provide the necessary statistical power, the recognition of the problems, the recognition of the risk of illness, the recognition of the need for preventive action, and the motivation for preventive action seemed related to the HLS-14 scores in both male and female and in both younger (35-44 years old) and older (45-59 years old) age groups.

Table 4 shows the comprehensibility of the health checkup report. Those who answered that the health checkup report was easy to read, understand, remember, locate the point, and keep for future reference accounted for $67.5,65.1,37.7,54.2$, and $65.3 \%$, respectively, in the study subjects. These percentages in the HLS-14 higher scoring group were significantly higher than those in the lower scoring group. As for the Lipkus-J, a significant difference between the higher and lower scoring groups was found in the difficulty of locating the point. As for the NVS-J, a significant difference between the higher and lower scoring groups was found in the difficulty of understanding and locating the point.

\section{Discussion}

Simply providing information is not enough to stimulate preventive action. It is vital to confirm that people can actually understand the information they need. Unfortunately, there have been no attempts to examine reading comprehension of personal health information like health checkup reports. This is the first study that determined the reading comprehension of health checkup reports in the context of HL in Japanese people. In the absence of established methodology, the assessment of reading comprehension was performed in terms of accomplishing the following fundamental purposes of health checkup reports: (1) recognition of the problems, (2) recognition of the risk of illness, (3) recognition of the need for preventive action, and (4) motivation for preventive action.

The items classified as abnormal were marked with signs clearly and were also mentioned in the doctor's advice in the health checkup report. Beyond our expectation, those who could correctly point out all the items classified as abnormal accounted for only $27.1 \%$ in the study subjects. More than one-third of the study subjects misread the normal/abnormal classification for the 7 items classified abnormal, respectively. The health checkup report used for the assessment represented a hypothetical case of metabolic syndrome that differed from the actual condition of the study subjects. The difference between hypothetical and actual data may confuse them and may affect their responses to some extent. However, it is worth noticing that quite a few examinees may misread the items classified as abnormal that require appropriate measures to minimize the risk of illness.

In spite of the misreading of health checkup results, about $75 \%$ of the study subjects agreed with the statements about the increased risk of illness and the need for preventive action for the examinee. Accordingly, most subjects expressed their willingness to take preventive action in the compliance with the doctor's advice after having received the health checkup report. These results do not support our hypothesis that only people who can identify their own problems will recognize their increased risk of illness and their need for preventive action, and consequently intend to take preventive action. Annual multiphasic health checkups have been firmly established in Japan, and people are likely to know full well that they should work at their health promotion subsequently to health checkups. Even though examinees cannot understand the details of health checkup results, they might have a vague sense that they must take appropriate measures to minimize their risk of illness.

Those who expressed their willingness to have a precise reexamination and a 6-month follow-up examination accounted for 64.2 and $61.6 \%$, respectively, which were relatively lower than that of improving current lifestyle (73.8\%) and having a regular checkup next year $(83.0 \%)$. This result is consistent with the findings in the national cancer screening programs that the percentages of examinees having a precise reexamination among those with abnormal findings ranged from $63.6 \%$ of colon cancer to $83.5 \%$ of breast cancer [15]. Many factors affect the likelihood that examinees with abnormal findings will revisit for diagnosis and follow-up. As mentioned above, part of the examinees who cannot understand the details of health checkup results will take preventive action, but the misreading of health checkup results may be one of the causes of lack of revisits.

The measurement of HL of the study subjects confirms our expectation that all examinees do not always have an adequate level of HL. In the analysis of the relationship between reading comprehension of health checkup reports and three types of HL measures, those with higher HLS-14 scores were significantly more likely to recognize the problems, the risk of illness, and the need for preventive action for the examinee, and also more likely to express their willingness to take preventive action in compliance with the doctor's advice after having received the health checkup report. Meanwhile, as for the Lipkus-J and the NVS-J, significant differences between the higher and 


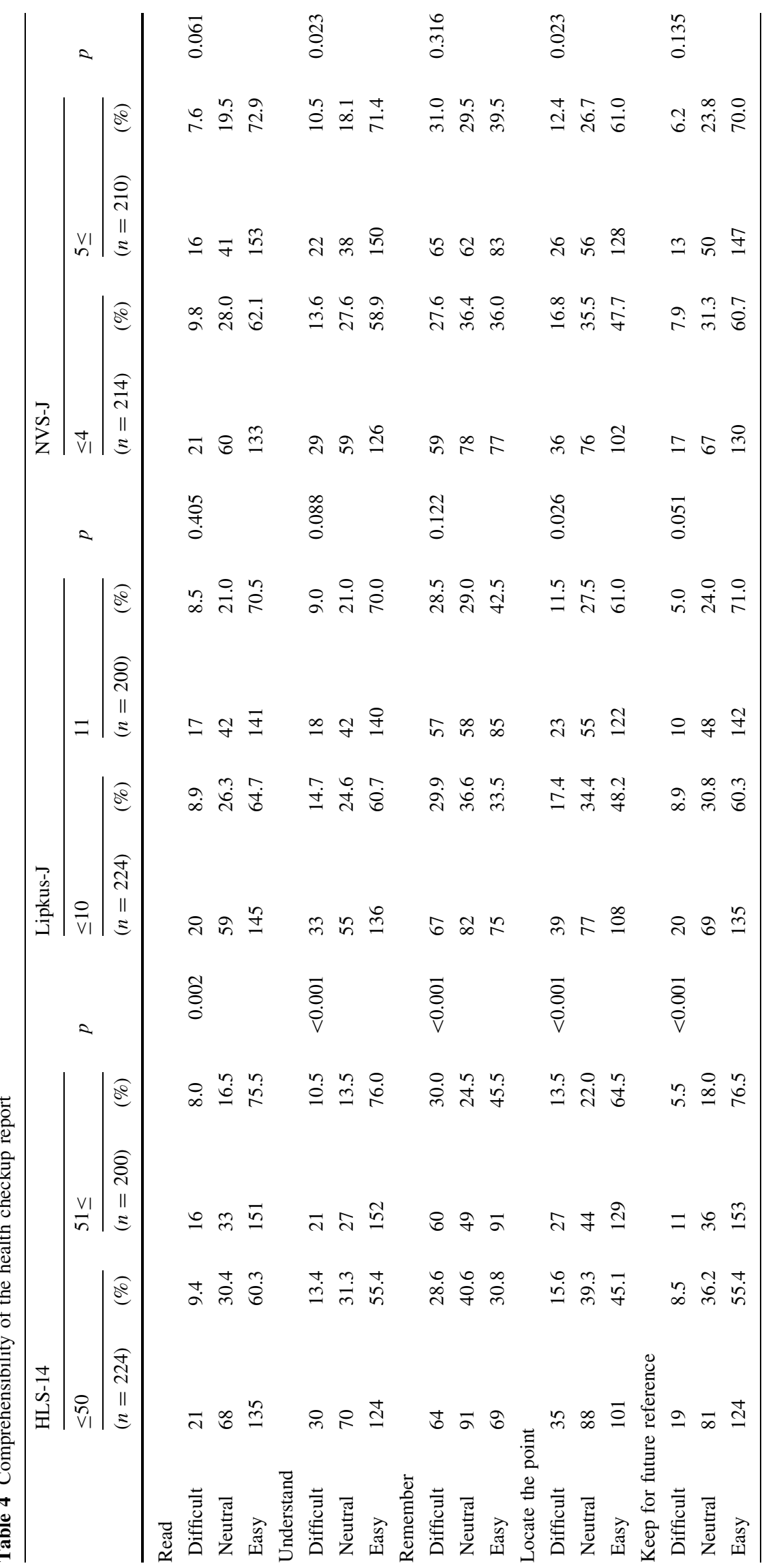


lower scoring groups were found in the recognition of the problems, but not in the recognition of the risk of illness, the recognition of the need for preventive action, or the motivation for preventive action, besides a few exceptions (the willingness to improve current lifestyle and have a regular checkup next year in relation to the NVS-J). The HLS-14 is a more comprehensive measure that covers all three levels of HL (functional, communicative, and critical) compared with the Lipkus-J and the NVS-J. The results of this study suggested that basic literacy and numeracy skills (i.e., functional HL) may contribute to the recognition of the problems, but more advanced skills (i.e., communicative $\mathrm{HL}$ and critical HL) are required for the recognition of the risk of illness, the recognition of the need for preventive action, and the motivation for preventive action.

Those who found no difficulty in reading understanding, remembering, locating, and keeping the health checkup report were more frequently observed in the HLS-14 higher scoring group. Those with adequate HL may be able to read their health checkup reports easily and utilize the information for their health promotion. Meanwhile, those with inadequate $\mathrm{HL}$ may have trouble in reading their health checkup reports and in some cases, may misread the information, which may affect their compliance with doctor's advice. Every examinee is notified of his/her health checkup results in written form according to the law in Japan. Further studies should be conducted to redesign the present health checkup reports to be understandable to all examinees regardless of their HL level.

This study provides the first step towards elucidation of the reading comprehension of health checkup reports in Japanese people. On the contrary, it has a number of potential limitations. First, the study subjects were selected from a nationwide panel of an online research company. Applicants for participation in the survey were accepted in the order of receipt until the number of participants reached the quotas. Unfortunately, we have no information about the number of subjects who would participate in the survey if we had not set the quota. As described in the "Results" section, the study subjects included highly educated people twice as many as in the Japanese population. Those who were more interested in health and more familiar with health information were likely to agree to complete the survey. The distribution of HLS-14 scores in the study subjects is quite similar to that obtained from our previous paper-based survey in a Japanese healthcare facility [6], but the selection bias may have influenced the results to some extent. Second, the web-based survey was self-administered, and thus the accuracy of responses must depend on their understanding of questions and their motivation to answer questions accurately. Although the understandability of wording were checked prior to the web-based survey, it is almost impossible to eliminate the information bias completely. Third, the method of assessing reading comprehension of health checkup reports was not fully validated. Similar performance-based assessments are commonly used for user-testing of written medicine and health information [16, 17]. The significant relationship between the reading comprehension of health checkup reports and the HLS-14 scores seems to support the validity of our assessment method, but further studies are needed to confirm the validity in other populations. Fourth, cross-sectional data were used, and thus causality cannot be inferred. Nobody doubts that HL is the major determinant of reading comprehension of health checkup reports. On the other hand, those who have read their health checkup reports may take an active interest in their health, which may enhance their HL level. It is possible that reading comprehension of health checkup reports and HL will be reciprocally enhanced. However, none of the three HL measures was significantly related to the frequency of health checkups in the study subjects. Health checkup experience seems to have only a limited effect on HL, if any.

\section{Conclusion}

Japanese people are supposed to have annual health checkups, and every examinee is notified of his/her health checkup results in written form. However, all examinees do not always have an adequate level of HL. After reading a specially designed health checkup report, about $70 \%$ of the study subjects misread the normal/abnormal classification for at least one items. Those with lower HLS-14 scores were significantly less likely to recognize the problems, the risk of illness, and the need for preventive action for the examinee, and also less likely to express their willingness to take preventive action in compliance with the doctor's advice after having received the health checkup report. HL may be the major determinant of reading comprehension of health checkup reports. For more effective health checkups, health promotion service providers should become aware of the existence of examinees with inadequate $\mathrm{HL}$ and address the problem of misreading health checkup results.

Acknowledgments This work was supported by the Research Grant from the Japan Society of Health Evaluation and Promotion and the Grant-in-Aid for Scientific Research (C) from Japan Society for the Promotion of Science (Grand Number 23590814).

Conflict of interests The authors declare that they have no conflict of interest.

\section{References}

1. Organization for Economic Co-operation and Development. Education at a Glance 2013: OECD indicator. Available at: http:// www.oecd.org/edu/eag.htm (Accessed 2014.2.1). 
2. World Health Organization. The WHO Health Promotion Glossary (WHO/HPR/HEP/98.1.) Available at: http://www.who.int/ healthpromotion/about/HPG (Accessed 2014.2.1).

3. Nutbeam D. Health literacy as a public health goal: a challenge for contemporary health education and communication strategies into the 21st century. Health Prom Int. 2000;15:259-67.

4. Ishikawa H, Takeuchi T, Yano E. Measuring functional, communicative, and critical health literacy among diabetic patients. Diabetes Care. 2008;31:874-9.

5. Ishikawa H, Nomura K, Sato M, Yano E. Developing a measure of communicative and critical health literacy: a pilot study of Japanese office workers. Health Promot Int. 2008;23:269-74.

6. Suka M, Odajima T, Kasai M, Igarashi A, Ishikawa H, Kusama $\mathrm{M}$, et al. The 14-item health literacy scale for Japanese adults (HLS-14). Environ Health Prev Med. 2013;18:407-15.

7. Okamoto M, Kyutoku Y, Sawada M, Clowney L, Watanabe E, Dan I, et al. Health numeracy in Japan: measures of basic numeracy account for framing bias in a highly numerate population. BMC Med Inform Decis Mak. 2012;12:104.

8. Kogure T, Sumitani M, Suka M, Ishikawa H, Odajima T, Igarashi A, et al. Validity and reliability of the Japanese Version of the Newest Vital Sign: a preliminary study. PLOS One. 2014 (in press).

9. Nakagami K, Yamauchi T, Noguchi H, Maeda T, Nakagami T. Development and validation of a new instrument for testing functional health literacy in Japanese adults. Nurs Health Sci. 2013 (in press).
10. Suka M, Odajima T, Sugimori H, Nakayama T. Evaluation of health checkup reports from consumer perspectives (in Japanese). Health Evaluat Promot. 2013;40:593-603.

11. Lipkus IM, Samsa G, Rimer BK. General performance on a numeracy scale among highly educated samples. Med Decis Making. 2001;21:37-44.

12. Barry DW, Mary ZM, William M, Kelley MC, Darren AD, Michael PP, et al. Quick assessment of literacy in primary care: the newest vital sign. Ann Fam Med. 2005;3:514-22.

13. Koo MM, Krass I, Aslani P. Evaluation of written medicine information: validation of the consumer information rating form. Ann Pharmacother. 2007;41:951-6.

14. Ministry of Internal Affairs and Communications. National Census 2010 (in Japanese). Available at: http://www.e-stat.go.jp/ SG1/estat/GL08020103.do?_toGL08020103_\&tclassID= $000001038689 \&$ cycleCode $=0 \&$ requestSender $=$ estat $\quad$ (Accessed 2014.2.1).

15. Ministry of Health, Labour, and Welfare. Report on Regional Public Health Services and Health Promotion Services 2011 (in Japanese). Available at: http://www.mhlw.go.jp/toukei/saikin/hw/ c-hoken/11 (Accessed 2014.2.1).

16. Luk A, Aslani P. Tools used to evaluate written medicine and health information: document and user perspectives. Health Educ Behav. 2011;38:389-403.

17. Raynor DK. User testing in developing patient medication information in Europe. Res Social Adm Pharm. 2013;9:640-5. 